Palabra Clave (La Plata)

ISSN: 1853-9912

palabraclave@fahce.unlp.edu.ar

Universidad Nacional de La Plata

Argentina

\title{
Política de Estado en edición de libros en la Norpatagonia: el Fondo Editorial Neuquino
}

\author{
Bertuzzi, Mauricio Carlos \\ Política de Estado en edición de libros en la Norpatagonia: el Fondo Editorial Neuquino \\ Palabra Clave (La Plata), vol. 9, núm. 2, 2020 \\ Universidad Nacional de La Plata, Argentina \\ Disponible en: http://www.redalyc. org/articulo.oa?id=350562513014 \\ DOI: https://doi.org/10.24215/18539912e087
}

Esta obra está bajo una Licencia Creative Commons Atribución-NoComercial-Compartirlgual 4.0 Internacional. 
Dossier: Catálogos y colecciones editoriales en Latinoamérica: abordajes teóricos, históricos y materiales

\title{
Política de Estado en edición de libros en la Norpatagonia: el Fondo Editorial Neuquino
}

Public book publishing policy in North Patagonia: The Neuquino Editorial Fund

Mauricio Carlos Bertuzzi

Universidad Nacional del Comahue, Argentina

mauricio.bertuzzi@gmail.com

\author{
DOI: https://doi.org/10.24215/18539912e087 \\ Redalyc: http://www.redalyc.org/articulo.oa? \\ $\mathrm{id}=350562513014$
}

Recepción: 04 Febrero 2020

Aprobación: 20 Marzo 2020

\section{Resumen:}

En el año 1989, la provincia de Neuquén crea el Fondo Editorial Neuquino (FEN) por Ley No 1.809 con el objetivo de publicar, difundir y vender obras de los escritores provinciales. Su puesta en funcionamiento y evolución fue dispar; a lo largo de los años tuvo diversas propuestas de modificación. La última de ellas, impulsada desde la Subsecretaría de Cultura (ahora con rango de Ministerio) concluyó a fines de 2018 en la redacción y aprobación de la Ley No 3.164, que la reemplaza.

Este proyecto editorial se solapó en sus 30 años de existencia con otros aportes de entidades estatales para publicar libros neuquinos, y coincidió en sus inicios con otras legislaciones provinciales similares en la Norpatagonia: en Río Negro surgió el Fondo Editorial Rionegrino en 1984, y un año después en La Pampa se reglamentó el Fondo Editorial Pampeano.

El presente trabajo describe y analiza el FEN poniendo de relieve particularidades de su redacción y puesta en funcionamiento. Busca discutir debilidades en su implementación, para contribuir al fortalecimiento de las estrategias públicas de edición de libros, considerando que la función del Estado para articular políticas activas es importante para promover e incrementar la bibliodiversidad del ecosistema libro argentino.

Palabras clave: Políticas públicas, Edición de libros, Bibliodiversidad, Fondo Editorial Neuquino, Argentina.

\section{Abstract:}

In 1989, the province of Neuquén created the Neuquino Editorial Fund (FEN, by its initials in Spanish) by Law number 1809 , with the aim of publishing, spreading and selling the works of local writers. The functioning and evolution of FEN was uneven, and there were several proposals to modify it over time. The last modification was sponsored by the Department of Culture (which today has the level of Ministry) in 2018, leading to the passing of Law number 3164, which replaced Law number 1809.

During its 30 years of existence, this editorial project overlapped with other contributions from state entities to publish books from Neuquén. In its beginnings it overlapped with other similar provincial laws in North Patagonia: the Río Negro province in 1984 created the Rionegrino Editorial Fund, and The Pampa province implemented the Pampeano Editorial Fund in 1985.

The present article describes and analyzes the FEN, highlighting the particular features of the way the law was drafted and implemented. It also aims to discuss some weaknesses in its implementation in order to help strengthen the state's strategies for book publishing, given the important role of the state in designing active policies to promote and increase the bibliodiversity of the Argentinean book ecosystem.

KEYWORDS: Public policies, Book edition, Bibliodiversity, Neuquino Editorial Fund, Argentina.

\section{INTRODUCCIÓN}

Hace 25 años que trabajo en temas relacionados con la edición de libros; en todas las instancias que participé descubrí el potencial comunicador y transformador que tiene el libro: "ese montón de hojas unidas por el lomo" que define el diccionario de la Real Academia Española. En la provincia de Neuquén hay un terreno fértil para la acción cultural, con una producción narrativa, poética y científica de cantidad y calidad superlativa que no tiene su correlato en la edición y circulación del que podríamos denominar "libro neuquino", aunque este concepto en sí mismo se preste a confusión y debate. Para los fines de este trabajo, acotaré el término al libro editado en la provincia de autor con vivienda en ella al momento de la publicación, 
aunque la ley analizada no lo defina claramente. En este sentido, el autor norpatagónico es desconocido fuera de un circuito cultural y académico muy acotado; por esto el investigador no puede ver reflejado su trabajo en los índices de citación de los mismos. El docente e investigador Ricardo Costa, por ejemplo, describe un periodo fértil en las letras neuquinas a principios de los ochenta, marcado por la creación del Centro de Escritores Patagónicos, la publicación de la revista Coirón, el grupo Poesía en trámite, lamentándose por su intransferencia al campo educativo (Costa, 2007).

En la Facultad de Humanidades de la Universidad Nacional del Comahue, una de las instituciones académicas más antiguas de la región, hay varios grupos de investigación dedicados a los estudios de la historia, el teatro y la literatura regionales. Ejemplo de ello son los Centros de Estudios de Historia Regional, Históricos de Estado, Política y Cultura, de las Dramaturgias de la Norpatagonia y el Grupo de Estudios de Historia Social. Pero estos estudios universitarios no hacen foco en la problemática de los libros y la edición aunque algunos trabajos del Centro Patagónico de Estudios Literarios, dirigido por Laura Pollastri -en especial los trabajos de Silvia Mellado- empiezan a mencionar el tema. La tesis doctoral de Mellado aborda la escritura en la Patagonia argentina y destaca que, hasta mediados de los años noventa, la literatura patagónica se pensaba en "el ámbito de los talleres literarios, los encuentros de escritores, los grupos poéticos o los colectivos de artistas..., y no (en) la academia...” (Mellado en Pollastri, 2014).

La gran cantidad de escritores e investigadores que producen textos en la región no tiene su correlato en la cantidad de editoriales con sede en la Norpatagonia, muy escasas y con poca o nula circulación de sus producciones. Una lectura atenta de los trabajos permite descubrir que la mayoría de las publicaciones tiene posibilidad de editarse a partir del apoyo estatal: aportes económicos a los autores, subsidios para la participación en eventos y, sobre todo, esfuerzos presupuestarios desde las áreas culturales municipales, provinciales y nacionales para la edición e impresión de libros. En tal sentido, en Neuquén aparecen muchas ediciones debido al aporte económico de la Dirección de Cultura Municipal, de concursos anuales promovidos por la Fundación del Banco de la Provincia del Neuquén y fundamentalmente a partir de la creación del Fondo Editorial Neuquino (FEN).

En el presente trabajo me propongo hacer foco en esta última política del Estado provincial para fomentar esta industria cultural, por ser la más extensa en el tiempo y porque en sus inicios coincide con otras legislaciones provinciales similares. En Río Negro surge el Fondo Editorial Rionegrino en 1984 mediante Ley provincial No 1.869 sancionada el 23/08 y promulgada el 04/09, y en La Pampa se reglamenta el Fondo Editorial Pampeano en 1985 (Decreto No 2.118 de Reglamentación de la Ley No 804).

No obstante, las tres políticas editoriales provinciales tienen sus características particulares, sus desarrollos han sido diferentes y merecerán un análisis comparativo posterior. En el caso particular del FEN su puesta en funcionamiento y evolución fue dispar; a lo largo de los años tuvo diversas propuestas de modificación. La última de ellas, impulsada por la Subsecretaría de Cultura de la provincia (ahora Ministerio de las Culturas) concluye a fines de 2018 en la redacción y aprobación de la Ley No 3.164, reglamentada por decreto el 3 de diciembre de 2019. De la misma participaron muchos de los actores y sectores involucrados en el ecosistema del libro de Neuquén: funcionarios provinciales, escritores y escritoras de las ciudades de Plottier, Centenario, Zapala, San Martín de los Andes, Villa La Angostura y Neuquén capital, representantes de editoriales independientes, organizaciones vinculadas a la difusión y promoción de las letras, entre otros.

Algunas preguntas que este trabajo empieza a formular son: ¿por qué un Estado, en este caso provincial, decide desarrollar una política editorial?; y ¿por qué motivo estas políticas estatales suelen interrumpirse, discontinuarse, o desviarse de los objetivos iniciales? Otros cuestionamientos tienen que ver con la producción y puesta en circulación de los libros editados a través de la convocatoria estatal. ¿Qué libros se seleccionan para ser editados?, ¿cómo es el proceso de selección?, ¿qué actores participan del mismo? Y una vez producidos, ¿cómo se ponen en circulación y se distribuyen los libros?

Abordar estos interrogantes es una tarea necesariamente interdisciplinaria, inscripta en los estudios sobre el libro y la edición que surgieron en los últimos años, a partir de la necesidad de desentrañar las 
particularidades de la producción, la circulación y el consumo de uno de los productos culturales más importantes y perecederos. A nivel internacional, son conocidos los trabajos sobre el tema de Roger Chartier (1994, 2016), Robert Darnton (1987, 2008a, 2008b), George Steiner, entre otros. En Argentina, son referentes de estos estudios José Luis de Diego (2006, 2017), Gustavo Sorá, Leandro de Sagastizabal (1995), Alejandra Giuliani y Alejandro Dujovne (2014), que desde el año 2012 dirige el Programa de Estudios del Libro y la Edición en el Instituto de Desarrollo Económico Y Social (IDES). Todos ellos, desde diversas disciplinas como la teoría literaria, antropología social, historia, sociología, abordan esta problemática.

\section{LA EDICIÓN DE LIBROS EN LA NORPATAgONIA}

A los fines de aproximación al tema, es posible reconocer un primer momento, que abarca desde los comienzos de la impresión de libros en la llamada "etapa territoriana" (Neuquén es reconocida como provincia desde 1955), hasta la sanción a fines de la década del ochenta de la ley de fomento editorial. En este primer periodo hay muy pocos casos de trabajo editorial y en el día a día, la labor de realizar libros la llevan a cabo los imprenteros. El libro Recuerdos territorianos de Ángel Edelman (1954) tiene dos capítulos destinados a enumerar las imprentas que existían en Neuquén a la fecha de su publicación y los periódicos que cada una de ellas editaba. Esto se debe a que las primeras imprentas instaladas en el territorio nacional del Neuquén estaban asociadas a la "militancia" -conservadora o liberal-y a la edición de un periódico y una guía comercial. El mismo Edelman era propietario de una imprenta, cuyo edificio donde funcionó fue declarado Monumento Histórico Municipal mediante Ordenanza No 7.097/95.

La búsqueda de documentación del trabajo editor en este periodo es complejo, debido al escaso registro legal de los libros y a que las publicaciones en su mayoría iban a imprimirse en Capital Federal.

El segundo momento, que interesa a los efectos del presente trabajo, abarca desde la coyuntura económica y social que desemboca en la sanción de la ley de fomento editorial a fines de los ochenta hasta la actualidad, donde los actores involucrados en el ecosistema libro neuquino participan de una serie de encuentros que confluyen en un proyecto de modificación de la ley que se sanciona a fines de 2018 y se reglamenta recientemente.

Abordo la lectura comparativa de las dos legislaciones, la que rigió y la que guiara la actividad del FEN, poniendo de manifiesto algunos errores relacionados con su implementación, buscando prevenir posibles problemas en el funcionamiento de la nueva editora que tiene previsto el inicio de su trabajo durante el 2020.

A los efectos del presente trabajo recurro a los ejemplares editados por el FEN disponibles en bibliotecas públicas y al registro que se hizo oportunamente de ellos en la Agencia Argentina del ISBN, ya que fue imposible dar con las actas u otro documento de la actuación del FEN, a pesar de que algunos de sus integrantes reconocieron haber registrado todas las reuniones y acciones realizadas en sus años de actividad hasta su virtual paralización en 2005.

\section{LA LEY 1.809 DE CREACIÓN DEL FEN}

En el año 1989 la Legislatura de la provincia de Neuquén sanciona con fuerza de ley la creación del FEN (Ley provincial 1.809). En su primer artículo define su objeto: publicar, difundir y vender "la obra intelectual de los autores del Neuquén, que se sirvan de la palabra escrita como medio de expresión” (art. 1).

Esta ley "crearía grandes expectativas entre la comunidad de escritores residentes en la provincia" (Costa, 2007, p. 104) aunque también generaba una gran controversia a lo largo de su puesta en funcionamiento, sobre todo a partir de la selección de obras que realizaba a lo largo de las convocatorias su Comisión Ejecutora.

El fondo tendrá un carácter "permanente" (art. 2) constituido por diversas fuentes de ingresos, sin especificar su monto: una partida del presupuesto, legados y donaciones, recursos provenientes de la gestión 
específica y de la venta de lo producido y los saldos de ejercicios anteriores. La redacción y puesta en práctica de este artículo fue uno de los obstáculos principales a la hora de poner en funcionamiento el proyecto editorial. También fue uno de los articulados que se sometió a mayor debate en el trabajo de redacción de la nueva ley sancionada el 5 de diciembre de 2018. La misma, precisa en su tercer artículo que el Poder Ejecutivo provincial asigne específicamente un aporte en el presupuesto de Cultura y este era "como mínimo (...) equivalente a diez aportes para los gastos de funcionamiento que la Comisión Nacional de Bibliotecas Populares (CONABIP) le otorga a las bibliotecas populares". Este aporte es variable, las bibliotecas populares lo reciben de varias maneras pero para gastos corrientes, según revistan categoría $\mathrm{A}, \mathrm{B}$ ó $\mathrm{C}$, asciende a $\$ 70.000$ promedio.

El principal cambio que operó en la ley que regula el FEN tiene que ver con la conformación de la Comisión que lo administra y hará también a partir de la nueva reglamentación. En 1989 se decidió que este aspecto y la selección de obras a publicar iba a estar a cargo de una comisión de 10 personas, conformada de la siguiente manera: 3 representantes de la Sociedad Argentina de Escritores (SADE), 2 de la Junta de Estudios Históricos de la provincia de Neuquén, 1 del Consejo de Planificación y Acción para el Desarrollo, 1 del Consejo Provincial de Educación, 1 del Sindicato de Prensa del Neuquén y 2 de la Federación de Bibliotecas Populares de Neuquén. La misma estaría presidida por el máximo cargo del área de Educación y Cultura de la provincia (en ese momento, el Subsecretario de Educación y Cultura). Los cargos eran ad-honorem y duraban un año (art. 3). Es posible vislumbrar a simple lectura las dificultades que tendría la Comisión para sesionar y acordar entre tan numerosa conformación. Así, al leer el articulado resuena la máxima atribuida a Napoleón y a Juan Domingo Perón: "Cuando quieras que algo no se haga, forma una comisión para hacerlo".

En la actualidad, la organización y la administración del FEN estará a cargo de un Consejo directivo presidido por un Director General designado por el Ministro de Cultura, un vocal propuesto por la misma área, 2 vocales en representación de los escritores y el/la subsecretario/a del área, ahora ministro, como representante institucional y legal (art. 4).

El cambio reduce la estructura administradora de diez a cuatro integrantes, pero le asigna la tarea de nombrar un jurado para la selección de las obras a publicar con el Fondo. Es decir que cada convocatoria deberá nombrar un jurado para evaluar y seleccionar lo que se vaya a publicar. Este cambio probablemente se deba a los problemas que tuvieron las anteriores comisiones para reunir a todos los integrantes; dificultad que señalaron en consultas informales y en las reuniones convocadas por la Subsecretaría de Cultura (ahora Ministerio) para debatir y consensuar la nueva ley. Un antiguo miembro del "sillón 10" del FEN lo señaló como principal causa del fracaso de las políticas editoriales del fondo.

Los siguientes artículos ( 5 al 8) describen y establecen las condiciones que tendrán que cumplir los autores para poder acceder a los beneficios del fondo, aunque le reserva a la Comisión la posibilidad de establecer excepciones y decidir en contrario.

El artículo 9 define los géneros que podrán publicarse: novela, cuento, teatro, poesía y críticas o ensayos (ya sean literarios, filosóficos, estáticos (sic), económicos, técnicos, sociales, históricos y políticos). La nueva redacción es más amplia ya que no define los géneros, aunque propone que las publicaciones los abarquen todos ("actuales y futuros") y exige que se alternen "para posibilitar una participación activa y renovada de la comunidad escritora" (art. 16).

En sus casi treinta años de existencia el FEN publicó 25 libros. Esto se desprende de la base de datos de la Agencia Argentina del ISBN (http://www.isbn.org.ar), es decir de los libros que tuvieron inscripción en este registro (International Standart Book Number). Los mismos abarcaron los siguientes géneros: poesía (7 libros, incluyendo la reedición de uno), cuentos (4), teatro (4), antologías de cuento y poesía (2), cuentos para niños (1), novela, relato y ensayo (3, 1 científico y 2 históricos) y catálogo de pintores (1). 
TABLA 1

Los libros editados por el FEN

\begin{tabular}{|c|c|c|c|}
\hline Título & Autor/es & Fecha & ISBN \\
\hline $\begin{array}{l}\text { Influencia del medio ambiente en la } \\
\text { expresión pictórica neuquina. }\end{array}$ & AA.VV. & sep-91 & $978-987-96044-0-3$ \\
\hline Cuentos 1991 & AA.VV. & dic-91 & $978-987-96044-7-2$ \\
\hline Imagen física de los sistemas cuánticos & Guala Valverde, Jorge A. & dic-92 & $978-987-96044-8-9$ \\
\hline Comarcas, aquí en el sur & Polito Belmonte, Santiago J. & dic-93 & $978-987-96044-2-7$ \\
\hline $\begin{array}{l}\text { La leyenda de El Dorado o Aguirre, el } \\
\text { marañón }\end{array}$ & Finzi, Alejandro & dic-94 & $978-987-96044-3-4$ \\
\hline Ajedrecias & AA.VV. & dic-94 & $978-987-96044-1-0$ \\
\hline Pioneros & Saccoccia, Hugo Luis & dic-94 & $978-987-96044-5-8$ \\
\hline Voz del desierto & Talero, Eduardo & dic-95 & $978-987-96044-4-1$ \\
\hline Neuquén & San Martín, Félix & sep-96 & $978-987-96044-6-5$ \\
\hline Comarcas, aquí en el sur & Polito Belmonte, Santiago J. & dic-97 & $978-987-9268-02-5$ \\
\hline Cuento-poesia '96 & AA.VV. & dic-97 & $978-987-9268-01-8$ \\
\hline Volver tus ojos & Peralta, María Cristina & dic -97 & $978-987-9268-00-1$ \\
\hline $\begin{array}{l}\text { El inmigrante. Cuentos vistos por el espejo } \\
\text { retrovisor }\end{array}$ & Pelliza, Sofía Delia M. & dic-97 & $978-987-96044-9-6$ \\
\hline La isla del fin del siglo & Finzi, Alejandro & $a b r-98$ & $978-987-9268-04-9$ \\
\hline Los caballos del verano & Pennini, Gerardo Leonardo & abr-98 & $978-987-9268-03-2$ \\
\hline Literatura infantil '98 y '99 & AA.VV. & nov-01 & $978-987-9268-10-0$ \\
\hline El Cartero. & Nordenstrom, Julio & nov-01 & $978-987-9268-09-4$ \\
\hline Poesía 1997-1998 & AA.VV. & nov-01 & $978-987-9268-08-7$ \\
\hline Cuento '97 y '98 & AA.VV. & nov-01 & $978-987-9268-07-0$ \\
\hline Las formas del Martín Fierro & Polito Belmonte, Santiago J. & nov-01 & $978-987-9268-06-3$ \\
\hline El raulí & Santos, Gustavo & nov-01 & $978-987-9268-05-6$ \\
\hline Cuento-poesía '99 & A.V.V. & abr-02 & $978-987-9268-14-8$ \\
\hline Veinticuatro letras & Brogna, Claudia Patricia & $a b r-02$ & $978-987-9268-13-1$ \\
\hline A cielo abierto & Lacava, Lidia Cristina & $\mathrm{abr}-02$ & $978-987-9268-12-4$ \\
\hline Obras inéditas & Lastra, Juan Julián & $a b r-02$ & $978-987-9268-11-7$ \\
\hline
\end{tabular}

Fuente: elaboración propia.

De estos libros, cuatro ediciones corresponden a obras que rescataron el patrimonio histórico de la provincia a través de textos fundacionales de la literatura neuquina (sus autores designan en la actualidad sendas calles de la ciudad de Neuquén), a saber:

- Voz del desierto, de Eduardo Talero (publicado por primera vez en 1907, de prosa elogiada por Jorge Luis Borges).

- Neuquén, de Félix San Martín (reedición del libro publicado por el Taller Gráfico de Luis Bernard en el año 1930 y con una edición previa en 1919).

- El cartero, de Julio Nordemstrom (como su subtítulo lo señala, las "memorias de un auténtico neuquino").

- Obras inéditas, de Juan Julián Lastra (recopilación de los poemas inéditos y escritos periodísticos del juez realizada por sus nietos).

El articulado de la ley le dio amplias facultades a la Comisión para decidir y actuar en materia editorial. Lo mismo hace la redacción actual, aunque divide la toma de decisiones, y se prevé un jurado integrado especialmente para cada convocatoria del FEN, el cual decidirá las obras a publicar. El mismo estará integrado por seis personas idóneas y de "reconocida trayectoria en el campo de la convocatoria" (art. 10): cuatro representando a cada una de las regiones de la provincia, uno en representación de las instituciones educativas o académicas que dictan carreras afines a Letras (universidades o institutos de formación docente) y otra persona perteneciente a la Junta de Estudios Históricos. En este sentido, cabe señalar que dicha Junta es la única institución que mantiene su representación en el Consejo directivo del FEN aunque al día de la fecha no tiene lugar físico para funcionar y han sido infructuosas las acciones por acceder a sus archivos (uno de los posibles lugares donde se encontrarían las actas del FEN). 
En cuanto a la tirada que tendrá cada publicación del FEN, ninguna de las dos redacciones de la ley lo establecen pero en el Art. 10 de la antigua ley, se especifica que los libros serán distribuidos de la siguiente manera: un $40 \%$ se dispondrán sin cargo (20\% para las bibliotecas populares, $5 \%$ para el autor, $5 \%$ para la prensa especializada, $5 \%$ para canje y $5 \%$ para el archivo). Si se tiene en cuenta que las bibliotecas populares de la provincia ascienden a ochenta y ocho, se puede estimar la tirada de los libros editados por el FEN en un mínimo de 500 ejemplares. En la publicación No 20: El raulí, de Gustavo Santos, se declara una tirada de 900 ejemplares. En cambio, los familiares de Julio Nordemstrom en nota periodística reconocen una tirada de 1000 ejemplares para el libro El cartero, edición No 16 del Fondo.

El nuevo articulado de la ley deja la decisión de definir la tirada en manos del Consejo directivo, atendiendo al "potencial de distribución y el impacto social" del tema (art. 18) aunque solicita un porcentaje no definido de la misma para distribuir sin cargo (art. 19).

Por último, en relación al destino de los fondos originados por la comercialización de los ejemplares, el Art. 11 de la "vieja" ley estipulaba que el autor percibiría el 10\% del precio de venta del ejemplar efectivamente comercializado pero renunciaría a su retribución en concepto de derechos de autor. En cambio, el nuevo articulado prevé un acuerdo individual con cada escritor del volumen a editar sobre sus derechos de edición y regalías, aunque le exige que registre su obra como inédita bajo la protección de la ley 11.723 de propiedad intelectual (arts. 21 y 22). Además, se deja constancia de que la editorial podrá disponer del material para publicarlo en otros formatos.

Lo anterior es la única referencia a uno de los temas fundamentales y más problemáticos de la industria editorial: la distribución y venta de los libros. Ninguna de las dos legislaciones establece la forma, el personal y el presupuesto que se tiene que destinar a la puesta a disposición del público de las publicaciones. Esta problemática de la circulación se agrava en el interior del país debido a los elevados costos de transporte. En las tres décadas que lleva funcionando el FEN, sus libros no encontraron un lugar en las vidrieras de las librerías. Una consulta informal con los dueños de las dos librerías tradicionales de la ciudad de Neuquén (Libracos y Logos) confirmó esta ausencia, ya que no recordaron haber tenido en exhibición y venta estos libros. Probablemente con la nueva ley tampoco se subsanará este problema.

\section{LOS PREMIOS DEL AÑO 2005}

A la fecha resultó imposible conseguir las Actas que registraron el accionar del FEN a lo largo de los años; la reconstrucción de las ediciones se realizó a partir de la base de datos de la Agencia Argentina del ISBN y de los ejemplares disponibles para consulta en las bibliotecas neuquinas, sobre todo la Biblioteca central de la Universidad Nacional del Comahue.

A partir de estos datos, es posible distinguir dos etapas en el trabajo del FEN: una que va desde su creación hasta el año 1997, cuando se produce un estancamiento y otra, entre 2001 y 2002, cuando se detiene nuevamente la edición de libros.

Pero en el año 2005, el FEN define a los ganadores de los concursos pendientes de los años 2000, 2001 y 2002, aunque hasta el día de la fecha no tienen publicación. La reconstrucción de este fallo es posible a partir una nota periodística aparecida en el diario Río Negro del día 11 de marzo de 2005, bajo el título "Premios del Fondo Editorial Neuquino" se informa que "la Secretaría de Cultura del Neuquén dio a conocer las listas de los ganadores de los concursos 2000, 2001 y 2002”. La información periodística da cuenta de premios en Poesía adultos 2000, Cuento Breve 2001 -adultos e infantil-, Novela corta e infantil 2002 y diversas menciones con un total de quince distinciones.

Los textos habían sido seleccionados por un jurado integrado por dos reconocidos docentes e investigadores en base a la preselección realizada por los escritores Marcelo Birmajer y Luis Tedesco, aunque no pudo constatarse que hayan pertenecido a la Comisión directiva del FEN. 
Estas discontinuidades y desaciertos en la aplicación de la ley $N^{\circ} 1.809$ llevaron a un trabajo de reformulación de la misma que tuvo sendos proyectos desde el año 2010, pero que recién se concretó a partir de las reuniones convocadas en 2017.

\section{LA NUEVA LEY $\mathrm{N}^{0} 3.164$}

El proyecto de nueva ley se consensuó durante dos jornadas de debate: la primera realizada en la ciudad de Neuquén el 27 de noviembre de 2017; la segunda, llevada a cabo en la ciudad de Zapala el 14 de mayo de 2018. Además, se abrió un foro virtual que recibió propuestas, comentarios y opiniones durante el año que duró el trabajo.

De estas reuniones participaron escritores y escritoras, representantes de editoriales independientes y entidades vinculadas a la difusión y promoción de las letras como SADE, funcionarios del área de Cultura, editores independientes, entre otros, que debatieron en varias mesas de trabajo los aspectos a tener en cuenta para la actualización de la ley. El trabajo produjo un anteproyecto que fue reelaborado por el equipo de la Subsecretaría de Cultura y un grupo asesor proveniente de la Legislatura neuquina; concluyó en la redacción de un proyecto de ley que fue aprobado y reglamentado por decreto el 3 de diciembre de 2019. El nuevo articulado derogó la Ley No 1.809 .

La insistencia en desarrollar una política editorial por parte del Estado provincial probablemente venga de la mano de la necesidad de generar espacios de interacción simbólica donde la ciudadanía, con una fuerte impronta de migración interna (en el pasado a causa de las grandes obras hidroeléctricas; en la actualidad, debido al "boom" petrolero), encuentre elementos en su historia -que en esta provincia es demasiado recientepara reconocerse como "neuquino". En este sentido, la ley No 3.164 se presentó junto a la ley No 3.163 que declaró patrimonio cultural inmaterial de la provincia las prácticas de las Cantoras Campesinas, una tradición centenaria que mantienen las mujeres del norte neuquino componiendo e interpretando tonadas, cuecas y décimas, "raspando" una guitarra criolla.

En la actualidad, el ahora Ministerio de las Culturas de la provincia trabaja en la formación del equipo de trabajo que relanzará el FEN. El funcionamiento futuro del mismo permitirá evaluar el cumplimiento de los objetivos propuestos en la Ley y, fundamentalmente, si el aporte estatal al ecosistema del libro argentino y su bibliodiversidad será sustancial o no.

\section{REFERENCIAS}

Costa, R. (2007). Un referente fundacional. Las letras neuquinas (periodo 1981-2005) y su (in)transferencia al campo educativo. Buenos Aires: El Surí Porfiado Ediciones.

Chartier, R. (1994). Elorden de los libros. Lectores, autores, bibliotecas en Europa entre los siglos XIV y XVIII. Barcelona: Gedisa.

Chartier, R. (2016). La mano del autor y el espiritu del impresor. Siglos XVI-XVIII. Buenos Aires: Katz Editores.

Darnton, R. (1987). La gran matanza de gatos y otros episodios en la historia cultural francesa. México: FCE.

Darnton, R. (2008a). ¿Qué es la historia del libro? Prismas: revista de historia intelectual, 12, 135-155.

Darnton, R. (2008b). Retorno a ¿Qué es la historia del libro? Prismas, revista de historia intelectual, 12, 157-168.

de Diego, J. (dir.) (2006). Editores y políticas editoriales en Argentina, 1880-2000. Buenos Aires: FCE.

de Diego, J. (2017). La otra cara de Jano. Una mirada crítica sobre el libro y la edición. Buenos Aires: Ampersand. de Sagastizabal, L. (1995). La edición de libros en la Argentina. Una empresa de cultura. Buenos Aires: Eudeba

Dujovne, A. (2014). Una historia del libro judio. La cultura judía argentina a través de sus editores, libreros, traductores, imprentas y bibliotecas. Buenos Aires: Siglo XXI editores. 
Edelman, A. (1954). Recuerdos territorianos, los primeros años de Neuquén capital. Buenos Aires: Talleres Gráficos de Juan Castagnola.

Giuliani, A. (2018). Editores y política. Entre el mercado latinoamericano de libros y el primer peronismo (1938-1955). Buenos Aires: Tren en Movimiento

Pollastri, L. et al. (2014). Los umbrales imposibles. General Roca: Publifadecs.

Sorá, G. (2017). Editar desde la izquierda en América latina. La agitada historia del Fondo de Cultura Económica y de Siglo XXI. Buenos Aires: Siglo XXI.

Steiner, G. (2009). Extraterritorial. Ensayos sobre la literatura y la revolución del lenguaje. Buenos Aires: Adriana Hidalgo.

\section{Fuentes documentales}

Diccionario de la Real Academia Española. http://www.rae.es

Premios del Fondo Editorial Neuquino. (2005). Diario Rio Negro. Recuperado de https://www.rionegro .com.ar/premios-del-fondo-editorial-neuquino-HYHRN0503113111004/

Foro abierto de actualización del Fondo Editorial Neuquino (FEN). Recuperado de https://culturaprov incia.neuquen.gob.ar/category/foro-abierto/

Ley provincial No 1.809/89. (1989) Recuperado de https://www.contadurianeuquen.gob.ar/ley-1809-1 989-creacion-del-fondo-editorial-neuquino-fen/

Ley provincial No 3.164/18. (2018). Recuperado de https://www.contadurianeuquen.gob.ar/ley-3164/

\section{BY-NC-SA}

